This is the author's final, peer-reviewed manuscript as accepted for publication. The publisher-formatted version may be available through the publisher's web site or your institution's library.

\title{
Single-molecule studies of disulfide bond reduction pathways used by human thioredoxin
}

Robert Szoszkiewicz

\section{How to cite this manuscript}

If you make reference to this version of the manuscript, use the following information:

Szoszkiewicz, R. (2013). Single-molecule studies of disulfide bond reduction pathways used by human thioredoxin. Retrieved from http://krex.ksu.edu

\section{Published Version Information}

Citation: Szoszkiewicz, R. (2013). Single-molecule studies of disulfide bond reduction pathways used by human thioredoxin. Biophysical Chemistry, 173-174, 31-38.

Copyright: (C) 2013 Elsevier B.V.

Digital Object Identifier (DOI): doi:10.1016/j.bpc.2013.01.002

Publisher's Link:

http://www.sciencedirect.com/science/article/pii/S030146221300015X

This item was retrieved from the K-State Research Exchange (K-REx), the institutional repository of Kansas State University. K-REx is available at http://krex.ksu.edu 


\title{
Single-molecule studies of disulfide bond reduction pathways used by human thioredoxin
}

\author{
Robert Szoszkiewicz ${ }^{a}$ \\ ${ }^{a}$ Department of Physics, Kansas State University, 307 Cardwell Hall, Manhattan, KS, USA. Fax: 785532 6806; Tel: 785-532-0855; \\ E-mail: szosz@ksu.edu
}

Disulfide bond reduction pathways used by human thioredoxin (hTrx) are studied at the single molecule level using a recombinant protein $\left(127_{\mathrm{SS}}\right)_{8}$. $\left(\mathrm{I} 27_{\mathrm{SS}}\right)_{8}$ contains eight tandem repeats of identical immunoglobulin-like modules with one disulfide bond in each module. Single $\left(\mathrm{I} 27_{\mathrm{SS}}\right)_{8}$ molecules are stretched at constant force applied by a cantilever in a force-clamp mode of atomic force microscopy (FC-AFM). Disulfide reduction events are accurately detected from stepwise increases in the end-to-end length of $\left(\mathrm{I} 27_{\mathrm{SS}}\right)_{8}$. Earlier FC-AFM studies observed one disulfide reduction pathway used by hTrx and suggested an additional electron tunnelling mechanism. Here, a very large set of unbiased FC-AFM data is collected in a range of clamping forces. By analyzing the data using exponential fits and dwell times histograms two disulfide reduction pathways used by hTrx are resolved. Based on previous studies one of these pathways is attributed to force-dependent Michaelis-Menten catalysis. The latter reduction pathway is weakly forceinhibited and occurs sporadically. Bimolecular nucleophilic substitutions $\left(\mathrm{S}_{\mathrm{N}} 2\right)$ and electron tunnelling (ET) mechanisms are discussed to explain the second pathway. Direct $\mathrm{S}_{\mathrm{N}} 2$ and ET mechanisms cannot be discounted, but a hypothetical E2-S $\mathrm{S}_{\mathrm{N}} 2$ mechanism involving a hydride reducing a disulfide bond provides an interesting alternative, which needs to be verified in future experiments.

\section{Keywords}

atomic force microscopy; single molecule biophysics; protein chemistry; disulfide bond reduction; thioredoxin; enzyme kinetics; ensemble kinetic analysis; dwell time histograms 


\section{Introduction}

Enzymatic catalysis outperforms man-made implementations for tightly controlled and stereo-selective chemical reactions with high yields. Molecular details of enzymatic catalysis are still largely unknown. Static protein structures, provided by cryo-electron microscopy NMR methods and X-ray crystallography, ${ }^{1-3}$ do not address enzymatic dynamics. Critical advancements are expected from dynamic NMR methods ${ }^{4-5}$ and single molecule methods. For example, recent force-clamp (FC) AFM studies reported on sub-Angstrom enzymatic reconfigurations in force-mediated catalysis of disulfide bond reduction by several kinds of thioredoxins. ${ }^{6-7}$ Thioredoxin is a ubiquitous disulfide bond reductase primarily responsible for cell redox homeostasis, antiapoptotic activity, and signaling. ${ }^{8,9}$

The FC-AFM studies of thioredoxin catalyses used a recombinant protein $\left(\mathrm{I} 27_{\mathrm{SS}}\right)_{8} \cdot{ }^{6,7,10} \mathrm{~A}\left(\mathrm{I} 27_{\mathrm{Ss}}\right)_{8}$ molecule contains eight identical modules of the $27^{\text {th }}$ domain from human cardiac titin with precisely one disulfide bond in each module. Each disulfide bond traps a portion of a protein chain, which unfolds after the bond is reduced. $\left(\mathrm{I} 27_{\mathrm{SS}}\right)_{8}$ is mechanically clamped between an AFM tip and an arbitrary surface, and reduction events are detected from increases in the protein end-to-end length, i.e., from the FC-AFM length vs. time trace. Using this method, disulfide reduction rate constants were obtained from single exponential fits to the FC-AFM length traces at a given force. In several types of Escherichia coli thioredoxins (TRX) disulfide reduction rate constants initially decreased and then increased as clamping force increased. ${ }^{6,7}$ In the case of hTrx, which is structurally similar to E.coli TRX, disulfide reduction rate constants decreased monotonically with force and remained constant above $300 \mathrm{pN}^{6}{ }^{6}$ This has been explained by molecular dynamics simulations, which obtained notable differences in width and depth of an enzymatic substrate binding groove as well as different mobilities of a substrate bound to prokaryotic vs. eukaryotic thioredoxins. ${ }^{7}$ Furthermore, electron tunnelling mechanism was suggested in thioredoxin catalysis. ${ }^{7}$

Overall, earlier experimental data and MD simulations found one disulfide reduction pathway in hTrx and suggested an additional electron tunnelling mechanism. To verify whether ET is a viable option and to address whether more than one disulfide reduction pathway is used by hTrx a large number of carefully analyzed FC-AFM data is needed.

Methods for the FC-AFM data analysis include exponential fitting to averaged length vs. time FC-AFM traces and dwell time analysis. ${ }^{11,12}$ Exponential fitting was applied extensively in ion channel literature ${ }^{24,25}$ and FC-AFM studies. ${ }^{6,10}$ Dwell time is the time elapsed from presenting a disulfide bond to a reducing environment to an actual bond rupture. Pioneered by Sigworth and Sine in patch clamp studies, dwell time histograms with a square root ordinate vs. logarithm of dwell times display peaks at each rate constant and their errors are distributed evenly for each bin. ${ }^{13-15}$ In a previous FC-AFM study, logarithmic dwell times histograms resolved two catalytic pathways for E.coli TRX reducing $\left(\mathrm{I}_{2} 7_{\mathrm{SS}}\right)_{8}$ at $100 \mathrm{pN}$ clamping force. ${ }^{10}$

In this manuscript, disulfide bond reductions by human thioredoxin are addressed using a large number of the FC-AFM data. To compare with previous studies $\left(\mathrm{I} 27_{\mathrm{SS}}\right)_{8}$ is used in a range of clamping forces from $100 \mathrm{pN}$ to $400 \mathrm{pN}$. Rate constants for disulfide bond reductions are obtained from exponential fits and dwell time analysis. The experimental data at $100 \mathrm{pN}$ and $200 \mathrm{pN}$ clamping forces are well fitted using two reaction rate constants, while the data at $300 \mathrm{pN}$ and $400 \mathrm{pN}$ are adequately fitted using one reaction rate constant. Thus, existence of two disulfide reduction pathways in hTrx is established, and particularly due to usage of dwell time histograms. One of the pathways is strongly force-inhibited and agrees with previously observed force-dependent Michaelis-Menten type of catalysis by thioredoxins. ${ }^{6,7,10}$ The latter pathway is very infrequent and weakly force-inhibited. Direct $S_{N} 2$ mechanisms and an electron tunnelling mechanism are thoroughly discussed to explain the latter pathway. While none of these mechanisms is discounted, they are not very likely in the light of the presented data and literature 
reports. Instead, a hypothetical E2-S $\mathrm{S}_{\mathrm{N}} 2$ mechanism involving a hydride reducing a disulfide bond provides an interesting alternative, which needs to be verified in future experiments.

\section{Materials and Methods}

2.1. Proteins and Enzymes - Expression and purification of $\left(\mathrm{I} 27_{\mathrm{SS}}\right)_{8}$ was described in detail previously. ${ }^{16}$ QuikChange site-directed mutagenesis using a kit from Stratagene introduced Gly32Cys and Ala75Cys mutations into each $\mathrm{I} 27$ module. Multiple rounds of $\mathrm{I}_{2} 7_{\mathrm{G} 32 \mathrm{C}-\mathrm{A} 75 \mathrm{C}}$ cloning created an N-C linked, eight-domain polyprotein gene of $\left(\mathrm{I} 27_{\mathrm{SS}}\right)_{8}{ }^{17}$ This gene was encoded in the vector pQE30 and expressed in the E. coli strain BL21(DE3). The pelleted cells were lysed by sonication and the His $_{6}$-tagged protein was purified using an immobilized Talon$\mathrm{Co}^{2+}$ column from Clontech followed by gel filtration with Supderdex 200 column from GE Healthcare. Purified protein was stored at $4^{\circ} \mathrm{C}$ in a buffer of $10 \mathrm{mM}$ HEPES, $150 \mathrm{mM} \mathrm{NaCl}, 1 \mathrm{mM}$ EDTA, $0.02 \% \mathrm{NaN}_{3}(\mathrm{w} / \mathrm{v})$, $\mathrm{pH}$ 7.2. Human thioredoxin was purified as described in detail previously. ${ }^{18}$ The pACA/hTrx plasmid was expressed in BL21(DE3) cells. Cell pellets were lysed using a French press and stirred with $7 \%$ w/v streptomycin sulphate. Protein was precipitated by adding ammonium sulphate to $85 \%$ saturation. Crude extracts were applied to a DEAE 52 column equilibrated with $50 \mathrm{mM}$ Tris- $\mathrm{HCl}, \mathrm{pH} 7.5,1 \mathrm{mM}$ EDTA and $0.1 \mathrm{mM}$ DTT. Thioredoxin was eluted with a $\mathrm{NaCl}$ gradient, concentrated, and purified with a Sephadex G-50 column equilibrated with $50 \mathrm{mM}$ Tris-HCl, pH 7.5, $1 \mathrm{mM}$ EDTA and 0.1 mM DTT. Proper fractions were concentrated and further purified using E.coli thioredoxin antibody affinity chromatography. The hTrx concentration was determined spectrophotometrically at $280 \mathrm{~nm}$ using a molar extinction coefficient of $8,050 \mathrm{M}^{-1} \mathrm{~cm}^{-1}$.

For FC-AFM studies a buffer containing $10 \mu \mathrm{M}$ hTrx, $10 \mathrm{mM}$ HEPES buffer, $150 \mathrm{mM} \mathrm{NaCl}, 1 \mathrm{mM}$ EDTA, $2 \mathrm{mM} \mathrm{NADPH}$, and $50 \mathrm{nM}$ of eukaryotic thioredoxin reductase from rat liver from Sigma-Aldrich was titrated with $1 \mathrm{M} \mathrm{NaOH}$ to $\mathrm{pH}$ 7.2. An excess of NADPH and a catalytic amount of thioredoxin reductase were necessary to maintain $\sim 98 \%$ of hTrx in an active, reduced form. ${ }^{19-20}$

2.2. Force-Clamp AFM Spectroscopy - A custom built AFM setup ${ }^{10}$ with a typical distance resolution in Z-axis of $0.2 \mathrm{~nm}$ and feedback time constant of a few ms was used. Commercial V-shaped silicon nitride MLCT-C cantilevers from Bruker, with normal elastic spring constants of $20 \mathrm{pN} / \mathrm{nm}$, were calibrated in liquid prior to each experiment by a thermal method. ${ }^{10,21}$ Purified protein was deposited on gold coated glass cover slides from Fisher Scientific.

Data acquisition and data analysis programs were custom developed for Igor Pro 5.0 from Wavemetrics. A following FC-AFM protocol was applied ${ }^{10}$. Initial tip-sample contact was maintained using AFM feedback for $1 \mathrm{~s}$ with a contact force of $-1 \mathrm{nN}$. Next, the contact force was reduced to $-100 \mathrm{pN}$ and maintained for $100 \mathrm{~ms}$ to mark an arbitrary zero extended end-to-end length of $\left(\mathrm{I} 27_{\mathrm{SS}}\right)_{8}$. The $\left(\mathrm{I} 27_{\mathrm{SS}}\right)_{8}$ molecule was then stretched at $190 \mathrm{pN}$ for $0.15 \mathrm{~s}$ in order to quickly expose the disulfide bonds to a bathing solution. Next, a given clamping force was set for $60 \mathrm{~s}$ to collect all the reduction events, and not the outliers only. Disulfide bond cleavage was not observed in the absence of $h \operatorname{Trx}{ }^{16}$. Any significant changes in the protein end-to-end length occur after the molecule is extended by at least tens of nanometers. Thus, kinetics of disulfide reduction is not expected to depend on water structure on the surface ${ }^{22}$, nor on any elasto-adhesive properties of an AFM tip and a substrate ${ }^{23}$. Disulfide reduction events occur after initial $\left(\mathrm{I}_{2} 7_{\mathrm{Ss}}\right)_{8}$ unfolding, so each reduction is spatially isolated by distances of several times the size of a single thioredoxin molecule. This way, the FC-AFM data with long clamping times are 
unbiased and without any hidden global constraints ${ }^{24}$.

2.3. Data analysis - For assessing data fits a standard Pearson (chi-square) test is used; $\chi^{2}=\Sigma_{i}\left(\left(y_{i}-f\left(x_{i}\right)\right) / \sigma\right)^{2}$, where $\Sigma_{i}$ sums over all data points, $y_{i}$ is an experimental value at i-th point, $f\left(x_{i}\right)$ is a value of a fitted function at ith point, and $\sigma$ is a standard deviation of the fit. To differentiate unambiguously between different models, a standard reduced chi-square, $\chi_{\text {red }}^{2}$, criterion is used. $\chi_{\text {red }}{ }^{2}=\chi^{2} /(N-c)$, where $N$ is the number of points and $c$ is the number of constraints. A good fit is found if $\chi_{\text {red }}^{2}<1$.

Statistical bootstrapping yields standard errors of disulfide reduction rate constants obtained by exponential fitting $^{25}$. To do so, a subset of $N$ FC-AFM traces is collected by drawing them with replacements from experimentally collected set of $N$ FC-AFM traces at each given force. Each subset is averaged and fitted with exponentials to obtain reaction rate constants. This procedure is repeated 2000 times to generate a distribution of reaction rate constants. Standard deviation of this distribution is attributed to a standard error of exponential fits at a given force.

Dwell time analysis yields disulfide reduction rate constants from fits to an ordinate $y$ of a logarithmic dwell time histogram. As in Ref. ${ }^{13} y=n_{i}^{1 / 2}=\left(N^{*} \delta x^{*} g(x)\right)^{1 / 2}$, where $n_{i}$ is the number of events in i-th bin, $N$ is the total number of events, $x=\ln (t)$ is a logarithmic abscissa, $t$ is time, $\delta x$ is a bin size, and $g(x)$ is a probability density function; $g(x)=\Sigma_{i} a_{i} \exp \left(x+\ln \left(k_{i}\right)+\delta x / 2+\exp \left(x+\ln \left(k_{i}\right)+\delta x / 2\right)\right)$, where $\Sigma_{i}$ sums over fitted reduction rate constants $k_{i}$, and $a_{i}$ is a fraction of $N$. Using $\sigma=0.5$ for each bin, $\chi_{\text {red }}^{2}$ analysis produced an optimum bin size $\delta x$ of 0.34 at $100 \mathrm{pN}$ clamping force, and this bin size was also used in other histograms.

\section{Results}

Figure 1 shows a schematic FC-AFM setup. Figure 2 shows that under clamping force $\left(\mathrm{I}_{2} 7_{\mathrm{SS}}\right)_{8}$ unfolds partially to present disulfide bonds to a bathing solution containing hTrx. At $190 \mathrm{pN}$ clamping force this initial (I27 $\left.7_{\mathrm{SS}}\right)_{8}$ unfolding yields $10.7 \pm 1.1 \mathrm{~nm}$ steps in an end-to-end length of the protein. Subsequent disulfide reduction events produce steps from $13.2 \pm 1.3 \mathrm{~nm}$ at $100 \mathrm{pN}$ to $15.1 \pm 1.4 \mathrm{~nm}$ at $400 \mathrm{pN}$. Thus, disulfide reductions in (I27 $\left.7_{\mathrm{SS}}\right)_{8}$ are easily distinguished from any other events.

Next, disulfide reduction rate constants are obtained using exponential fitting to the reduction parts of FCAFM length traces, see Fig. 2. First, reduction parts of all FC-AFM length traces at a given clamping force are arranged to begin at an arbitrary zero length and then averaged. Fig. 3 plots the averaged data, which are fitted with single and double exponentials. Exponential fits yield the cumulative time constants $\tau_{i}$, which are related to cumulative reaction rate constants $r_{i}$ expressed in $\mathrm{s}^{-1}: r_{i}=1 / \tau_{i}$. Protein concentration is constant, since only one protein molecule with up to eight disulfide bonds is stretched at a time. This is why $r_{i}$ are pseudo-first-order rate constants. They are cumulative, since each $r_{i}$ results from a rate limiting step in a priori unknown series of reactions. These values of $r_{i}$ are called later "disulfide reduction rate constants". To quantify whether single or double exponential fits are better, fit residuals, $\chi_{\text {red }}{ }^{2}$ values, and standard errors of fitted rate constants are compared. See Supplementary materials for details. The results yield that at $100 \mathrm{pN}$ and $200 \mathrm{pN}$ clamping forces two exponential fits are necessary, while at $300 \mathrm{pN}$ and $400 \mathrm{pN}$ single exponential fits are adequate, i.e., single exponentials produce results comparable with double exponential fitting.

In addition, disulfide reduction rate constants are calculated using dwell times of reduction events ${ }^{10}$. Fig. 4 
displays logarithmic histograms of sorted dwell times from all FC-AFM length traces at a given clamping force. Logarithmic histograms are fitted with appropriate probability density functions, pdfs. See Materials and methods and Supplementary materials for details. First, rate constants are obtained by fitting pdfs without any assumptions. Next, one rate constant is constrained to be $0.1 \mathrm{~s}^{-1}$ across all clamping forces, and the other one is fitted. The value of $0.1 \mathrm{~s}^{-1}$ comes from unconstrained fits at $400 \mathrm{pN}$ clamping force, i.e., where disulfide reductions at $0.1 \mathrm{~s}^{-1}$ are dominant. Fits to the constrained logarithmic histograms turn out significantly worse than to the unconstrained histograms. This means that a second rate constant must depend on force. The $\chi_{\text {red }}{ }^{2}$ analysis and errors of the fitting parameters indicate that two reduction rate constants reproduce the data best at $100 \mathrm{pN}$ and $200 \mathrm{pN}$ clamping forces, while one reduction rate constant produces an adequate fit at $300 \mathrm{pN}$ and $400 \mathrm{pN}$ clamping forces.

The results from exponential analysis and dwell time histograms agree to resolve two disulfide reduction pathways, called later path A and path B. Disulfide rate constants for path A are $1.67 \pm 0.10 \mathrm{~s}^{-1}$ at $100 \mathrm{pN}$ and 0.54 $\pm 0.21 \mathrm{~s}^{-1}$ at $200 \mathrm{pN}$. Disulfide rate constants for path $\mathrm{B}$ are $0.20 \pm 0.03 \mathrm{~s}^{-1}$ at $100 \mathrm{pN}$ and $0.18 \pm 0.04 \mathrm{~s}^{-1}$ at $200 \mathrm{pN}$. At $300 \mathrm{pN}$ and $400 \mathrm{pN}$ paths $\mathrm{A}$ and $\mathrm{B}$ merge into one apparent pathway with rate constants of $0.10 \pm 0.01 \mathrm{~s}^{-1}$ at $300 \mathrm{pN}$ and $0.12 \pm 0.01 \mathrm{~s}^{-1}$ at $400 \mathrm{pN}$. Figure 5 presents these results.

\section{Discussion}

Disulfide bond reductions by thioredoxin in ensemble experiments were explained by an $\mathrm{S}_{\mathrm{N}} 2$ mechanism ${ }^{8,19}$. The $\mathrm{S}_{\mathrm{N}} 2$ mechanism alone, however, was not able explain non-monotonic force dependency of the reaction rate constant observed by FC-AFM in E.coli TRX, where two pathways were proposed ${ }^{6,7}$.

A predominant disulfide reduction pathway, Path I, found both in E.coli TRX and hTRX was explained by a Michaelis-Menten reaction with a force-decelerated catalytic rate constant ${ }^{6,7}$. This mechanism involves initial substrate - enzyme binding followed by actual catalysis. An initial binding step was found to be much faster than a catalytic step, because prior to catalysis the protein's disulfide bond had to rotate to achieve co-linearity of three sulfur atoms involved in the reaction. Beyond two sulfur atoms forming a disulfide bond, the third atom is one of the two electron providing sulfur atoms in a thioredoxin catalytic center. As verified by molecular dynamics (MD) simulations, the reaction promoting disulfide bond rotation shortened its length projected upon a pulling axis. Increase of tensile force decreased efficiency of Path I due to increased difficulty for an enzyme to rotate a disulfide bond at elevated forces.

At elevated clamping forces Path II was proposed to dominate in E.coli $\mathrm{TRX}^{6,7}$. Path II was explained by a straightforward $\mathrm{S}_{\mathrm{N}} 2$ mechanism, which does not involve any canonical substrate-enzyme binding, but occurs via spontaneous collisions of thioredoxin with a stretched protein. Successful collisions are not very frequent, but at increased tensile force a disulfide bond in $\left(\mathrm{I} 27_{\mathrm{SS}}\right)_{8}$ is closer to a transition state for its reduction. Recent MD simulations showed that loose protein-thioredoxin associations were likely in E.coli TRX due to its shallow substrate binding groove and large substrate mobilities in the groove ${ }^{7}$. However, a substantially deeper substrate binding groove in hTrx than in E.coli TRX would prohibit Path II in hTrx, which agreed with the experimental data $^{7}$.

Furthermore, based on non-specific disulfide reductions of $\left(127_{\mathrm{SS}}\right)_{8}$ by $\mathrm{Zn}$ nanoparticles and its independence on concentration of $\mathrm{Zn}$ nanoparticles, a ubiquitous electron tunnelling mechanism of disulfide reduction was stipulated to be exercised by all kinds of thioredoxins ${ }^{7}$. 
Overall, earlier experimental data and MD simulations found one disulfide reduction pathway, Path I, in hTrx, and suggested an additional electron tunnelling mechanism. Our experimental results provide a direct experimental evidence for two disulfide reduction pathways, paths A and B, in hTrx, and particularly so by usage of dwell time histograms.

To start data analysis, we note that path $\mathrm{A}$, which is prevalent at $100 \mathrm{pN}$ and $200 \mathrm{pN}$ clamping forces is likely related to Path I from earlier studies, because hTrx and $\left(\mathrm{I} 27_{\mathrm{SS}}\right)_{8}$ used by Wiita et al. ${ }^{6}$ are used here too. Due to potential differences in enzymatic activity and differences in methodologies between the study of Wiita et al. and a current study, the absolute values of the corresponding rate constants are not compared directly. Instead, to test whether path A and Path I are related, a ratio, $R$, is calculated, where $R=$ (a rate constant for path $\mathrm{A}$ at 100 $\mathrm{pN}) /($ a rate constant of path $\mathrm{A}$ at $200 \mathrm{pN})$. Using current results one gets $R=3.4 \pm 0.6$ from logarithmic histogram fitting and $R=3.1 \pm 1.3$ from exponential fits. The model of Wiita et al. yields $R=3.4 \pm 0.5$. Consequently, path $\mathrm{A}$ is attributed to a force-decelerated Michaelis-Menten disulfide reduction pathway, see Scheme 1. Since mechanism details of path A are well established ${ }^{6,7}$, mechanistic details of path B are discussed.

A direct force-dependent pseudo-first order reaction mechanism ${ }^{6,7}$ is described by a rate constant $k_{02}$, which depends on two parameters $\gamma$ and $\Delta x_{02}$, see Scheme $1 ; \gamma$ is a second order rate constant, and $\Delta x_{02}$ is an averaged distance a disulfide bond must stretch along a reaction coordinate to reach a transition state. For best precision in obtaining $\gamma$ and $\Delta x_{02}$ a linear fit to a natural logarithm of $k_{02}$ vs. clamping force is produced, see Supplementary materials, to yield $\gamma=24 \pm 7 \mathrm{nM}^{-1} \mathrm{~s}^{-1}$ and $\Delta x_{02}=-9 \pm 4 \mathrm{pm}$. Thus, using a large number of data acquired in a current study we are able to show that path B is only weakly force-decelerated, i.e., with a small and negative $\Delta x$.

Theoretical and experimental single molecule studies of disulfide reductions by small inorganic molecules obtained that a direct mechanism, e.g., a $\mathrm{S}_{\mathrm{N}} 2$ mechanism, would produce strongly force-accelerated disulfide reductions with $\Delta x$ of $30-40$ pm, i.e., corresponding to $20 \%$ stretching of a -S-S- bond from its initial length of $205 \mathrm{pm}$ to $240 \mathrm{pm}$ at a transition state ${ }^{16,26}$. However, in recent FC-AFM experiments a transition from $\Delta x \sim 40-$ $50 \mathrm{pm}$ to $\Delta x \sim 5-15 \mathrm{pm}$ was observed above $500 \mathrm{pN}$ clamping force ${ }^{27}$. Sudden decrease of $\Delta x$ at elevated clamping forces was interpreted as "cis" to "trans", or "boat" to "chair", conformational switch of a disulfide bond prior to its reduction. Quantum calculations show that a "cis" to "trans" transition extends the disulfide bond by about $10 \mathrm{pm}$, which brings it closer to a transition state for a subsequent disulfide reduction ${ }^{27,28}$. Furthermore, the "trans" arrangement of -S-S- atoms makes them accessible for an additional "backside" $\mathrm{S}_{\mathrm{N}} 2$ disulfide reduction mechanism. While the force range exploited here does not extend to $500 \mathrm{pN}$, some rare "cis" to "trans" rearrangements of disulfide bonds would be expected to occur at lower forces too. This scenario would account on small values of $\Delta x$ for the path $\mathrm{B}$, but to the best of the author's knowledge, quantum calculations, e.g., density functional theory calculations, substantiating such a view have not been published yet.

Path B could also relate to electron tunnelling ${ }^{7}$. Tunnelling involves no bond rupture, no energy barrier, and hence no transition state ${ }^{29}$. There is, however, a reorganization energy barrier is involved in ET, since the system must prepare by obtaining reactive radicals. Here, the ET process is likely to proceed via a thiyl radical ($\mathrm{S}$ ) in one of the a catalytic cysteines and a subsequent electron transfer to a disulfide bond in $\left(\mathrm{I} 27_{\mathrm{SS}}\right)_{8}$. Then, the disulfide reduction itself proceeds via a thyil radial and a thiolate $\left(-S^{-}\right)^{30}$. Formation of a thyil radial and a thiolate breaks open the disulfide bond, which is then separated by mechanical force. Using Marcus theory of electron tunnelling together with recent derivations of Moser-Datton and other authors ${ }^{31-33}$ we calculate in detail in the $\underline{\text { Supplementary materials that tunnelling rate constants of the order of } 10^{3} \mathrm{~s}^{-1} \text { are expected in the case of a sketched }}$ 
electron tunnelling mechanism. Such rate constants of $10^{3} \mathrm{~s}^{-1}$ are two orders of magnitude larger than what is possible to measure using current FC-AFM setups ${ }^{36}$. Thus, while electron tunnelling cannot be excluded, its experimental verification is expected to involve FC-AFM spectrometers with much higher bandwidth.

A hypothetically suitable mechanism of path B could proceed as follows. A bimolecular elimination, E2, is known to compete with an $\mathrm{S}_{\mathrm{N}} 2$ mechanism, and particularly in the case of an inaccessible nucleophile. In the most likely E2 scenario a negatively charged sulfur atom from one of the catalytic cysteines would attack a $\beta$-carbon in one of the cysteines in a disulfide bond. Similarly to an $S_{N} 2$ mechanism an E2 mechanism occurs in one step, so at the same time a single bond between an $\alpha$-carbon and a $\beta$-carbon in a cysteine residue becomes a double bond. This is a preferred strategy based on Zaitsev's rule governing E2 reactions, i.e., it creates a more substituted alkene than a terminal $\mathrm{S}=\mathrm{C}$ - configuration, which would arise from a rupture of a disulfide bond, see Scheme 1 . A double bond between an $\alpha$-carbon and a $\beta$-carbon in a cysteine residue requires a hydride, $\mathrm{H}^{-}$, to leave the $\alpha$-carbon. The other hydrogen would leave in a form of hydron, $\mathrm{H}^{+}$, to form a terminal - $\mathrm{SH}$ group in originally attacking catalytic cysteine of hTrx, see Scheme 1. Hydrides are bad leaving groups due to being strong bases. However, organic chemistry books show many examples of leaving hydrides. For example, hydrides can move between neighboring carbons to generate more stable carbocations. Here, a hydride is hypothesized to be generated by leaving a betacarbon in one of the cysteines within I27. A hydride is a nucleophile and can attack a disulfide bond to be broken, see Scheme 1. Hydrides, however, are very unstable in solution, so that a subsequent nucleophilic $\mathrm{S}_{\mathrm{N}} 2$ attack on a disulfide bond would occur, because the -S-S- bond is only several Ångströms away.

The current data does not warrant any claim of demonstrating any novel disulfide reduction mechanism used by hTrx. Stringent experimental verification, i.e., at different buffers or using isotope effects, is needed. However, there are several advantages of a proposed E2 mechanism with subsequent $S_{\mathrm{N}} 2$-type nucleophilic attack of a hydride on a disulfide bond. First, each $\beta$-carbon in cysteine residues within $\left(\mathrm{I}_{2} 7_{\mathrm{SS}}\right)_{8}$ is surrounded by two hydrogens, and thus quite exposed for a nucleophilic attack by thioredoxin. Second, a hydride is small and a disulfide bond is easily accessible for it. Third, this mechanism explains small, force-decelerated, rate constants. This is because any nucleophilic attack of $\mathrm{H}^{-}$on a sulfur atom in a disulfide bond becomes less probable when bonds are elongated. Probability of this process depends on a volume, which a hydride needs to explore before reaching a sulfur atom. Excluding any electrostatic guiding ${ }^{37}$, this volume is a sphere with a radius $r_{H t o S}$, i.e., a distance between a hydrogen at an $\alpha$-carbon and its nearest sulfur atom in a disulfide bond to be broken. Supposing $\sim 10 \%$ increase in $r_{H t o S}$, when force changes from $100 \mathrm{pN}$ to $400 \mathrm{pN}$, one obtains about $30 \%$ decrease in the rate constant, which explains the experimental data. While $10 \% r_{H t o S}$ increase of $r_{H t o S}$ is reasonable based on MD simulations of a disulfide bond stretching ${ }^{6,26,27}$, the E2-S $\mathrm{S}_{\mathrm{N}} 2$ mechanism is currently only a possibility, which needs to be verified using further studies. Please note, however, path A is equally elaborated, since it requires twisting of a disulfide bond before reducing it.

Finally, potential conformational flexibility of hTrx might relate to mechanistic subtleties. By using available structural data of TRX bound to three different polypeptides Wiita et al. ${ }^{6}$, obtained via molecular dynamics simulations distinctively different reaction rate constants for path $\mathrm{A}$ in the case of each peptide. Different reaction rates where associated with different binding ways of each peptide. Any statistically justifiable spread in binding conformations between hTrx and a disulfide bond embedded within I27 should result in a small spread of obtained reaction rates. In all single molecule assays with thioredoxin, however, a bimodal distribution of rates is observed ${ }^{6,7,10}$. Thus, thioredoxin-peptide conformations seem to be quite unique for each system. 


\section{Conclusions}

Due to a low reduction potential of thiols and their ease to be oxidized to sulfur species, disulfide chemistry is very rich, which can wreak havoc in tightly spaced cellular compartments if not properly controlled ${ }^{7,34,35,37,39}$. Through deeper binding groove and stronger substrate binding than E.coli analogs, human thioredoxin has evolved to achieve substantial enhancement of one major disulfide reduction pathway, and marginalization of other competing pathways ${ }^{7}$. This is a good strategy to enhance specificity of an enzyme. The FC-AFM studies at high clamping forces, however, are able to reveal marginalized disulfide reduction pathways.

Using a large set of FC-AFM data catalytic behavior of human thioredoxin in a force range between 100 $\mathrm{pN}$ to $400 \mathrm{pN}$ was analyzed here. Fits to an ensemble average of all FC-AFM length traces and dwell time histograms at a given force yielded disulfide reduction rate constants. This way two distinctive disulfide reduction pathways, A and B, were directly resolved. The paths A and B reflect dynamic variability of hTx enzymatic activity, which becomes experimentally accessible only when lots of experimental data is collected and analyzed with several data analysis methods.

Based on agreement between our results and previously published studies, path A was attributed to the Michaelis-Menten catalysis. The second, much less frequent, reaction pathway showed to be very weakly force dependent. Three possible mechanisms for path B were carefully discussed: a straightforward $\mathrm{S}_{\mathrm{N}} 2$ mechanism, electron tunnelling, and a combination of E2 with subsequent $\mathrm{S}_{\mathrm{N}} 2$ attack by a hydride. Direct $\mathrm{S}_{\mathrm{N}} 2$ and ET mechanisms cannot be discounted, but a currently hypothetical E2- $\mathrm{S}_{\mathrm{N}} 2$ mechanism provides an interesting alternative to explain the experimental data. However, stringent experimental verifications are needed, i.e., by using different buffers or isotope effects, to warrant such a E2-S 2 mechanism.

\section{Acknowledgments}

The experimental results presented in this paper were obtained in the labs of Prof. Julio Fernandez at Columbia University, NY, USA. Generous help and support of Prof. Fernandez for this project is greatly appreciated. We are also grateful to Dr. Carmelu Badilla for her invaluable help with protein biochemistry and for the expression of (I27SS)8 protein. We thank Prof. Arne Holmgren for providing human thioredoxin and Dr. Rodriguez-Larrea for thioredoxin purification. 


\section{Graphics}

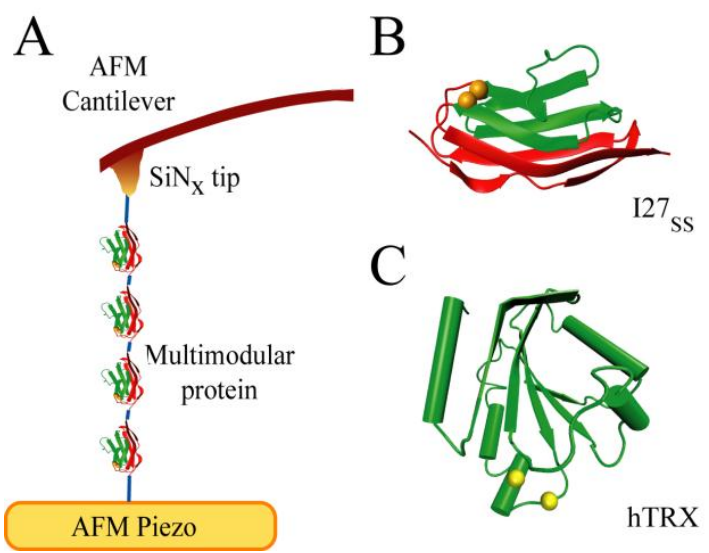

Figure 1. Experimental schematics. A) In the FC-AFM setup, a cantilever picks up, extends and keeps under a constant force a single molecule. B) Each module of (I27SS)8 is composed of an I27th domain of human cardiac titin (pdb: 1TIT) with one disulfide bond formed between residues 32 and 75 (yellow spheres). The residues in green are mechanically "trapped" behind the disulfide bond, while the residues in red unfold readily under force. C) Human thioredoxin (pdb: 3TRX). Yellow spheres represent $\alpha$-carbons of catalytic cysteines (Cys 32 and Cys 35) within an enzymatic center. 

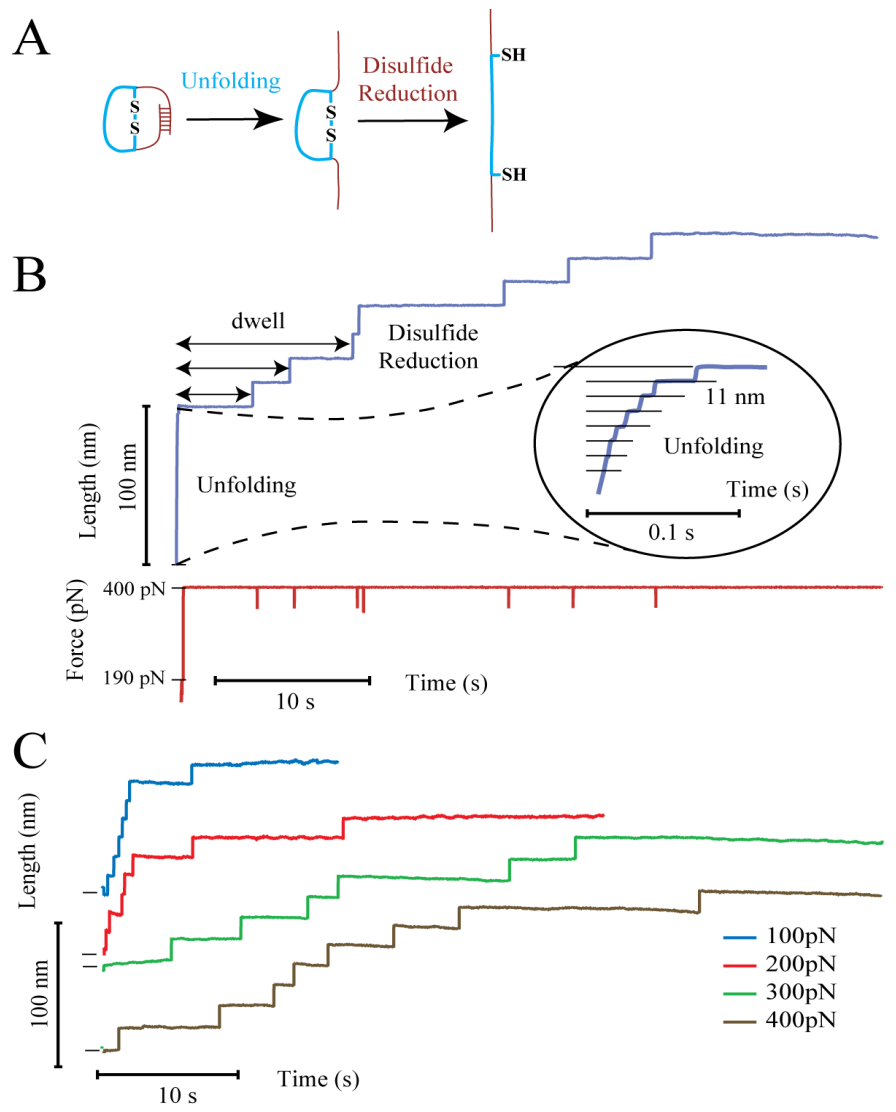

Figure 2. Sample experimental data. A) Under force, each module of $\left(\left[27_{\mathrm{ss}}\right)_{8}\right.$ partially unfolds and exposes its disulfide bonds to a bathing solution. Upon disulfide reduction unfolding of a remaining protein part takes place. B) An FC-AFM length trace measures end-to-end extended protein length vs time. At initial $150 \mathrm{~ms}$ at a pulling force of $190 \mathrm{pN}$ the protein extends elastically and partially unfolds. Then, pulling force is changed to an arbitrary value to study kinetics of disulfide bond reductions. The time between the beginning of a disulfide reduction phase and any of the disulfide reduction events is called the dwell time. First three dwell times are marked on a FC-AFM trace. Inset: At least six unfolding steps are resolved. C) A collection of four FC-AFM traces showing only a disulfide reduction phase, i.e., without an initial unfolding part. Each trace was recorded at different pulling forces. 

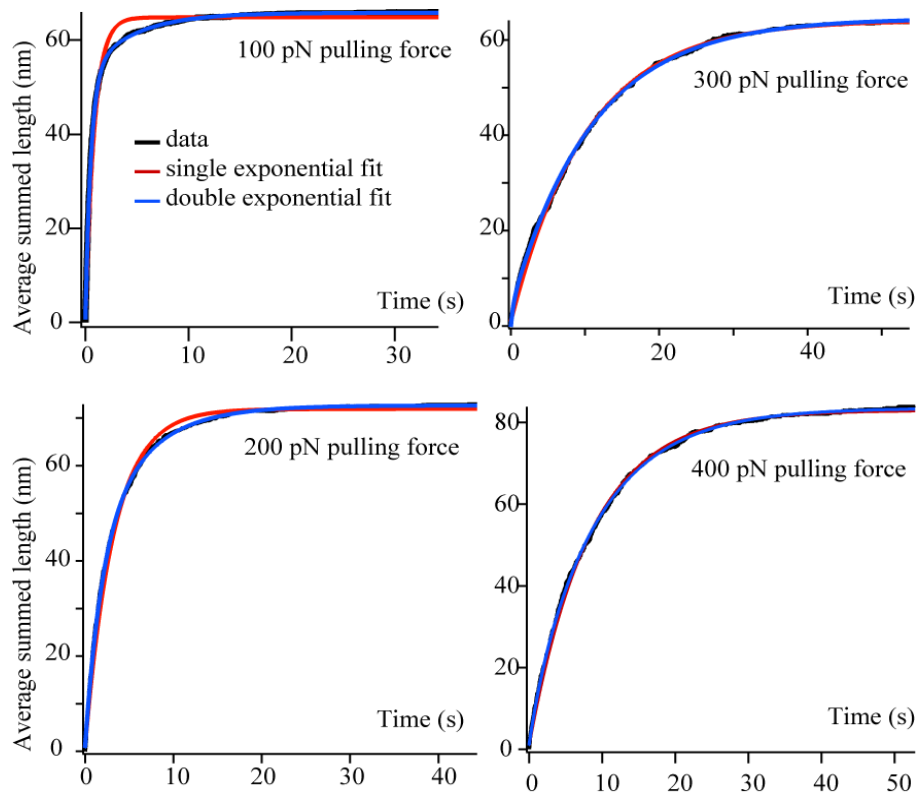

Figure 3. Analysis of disulfide reductions via exponential fits. Disulfide reduction rates are obtained by fitting single and double exponentials to an averaged FC-AFM length trace obtained after averaging all FC-AFM length traces collected at a given force. Double exponential fits perform well at $100 \mathrm{pN}$ and $200 \mathrm{pN}$, while single exponential fit are adequate at 300 pN and $400 \mathrm{pN}$.
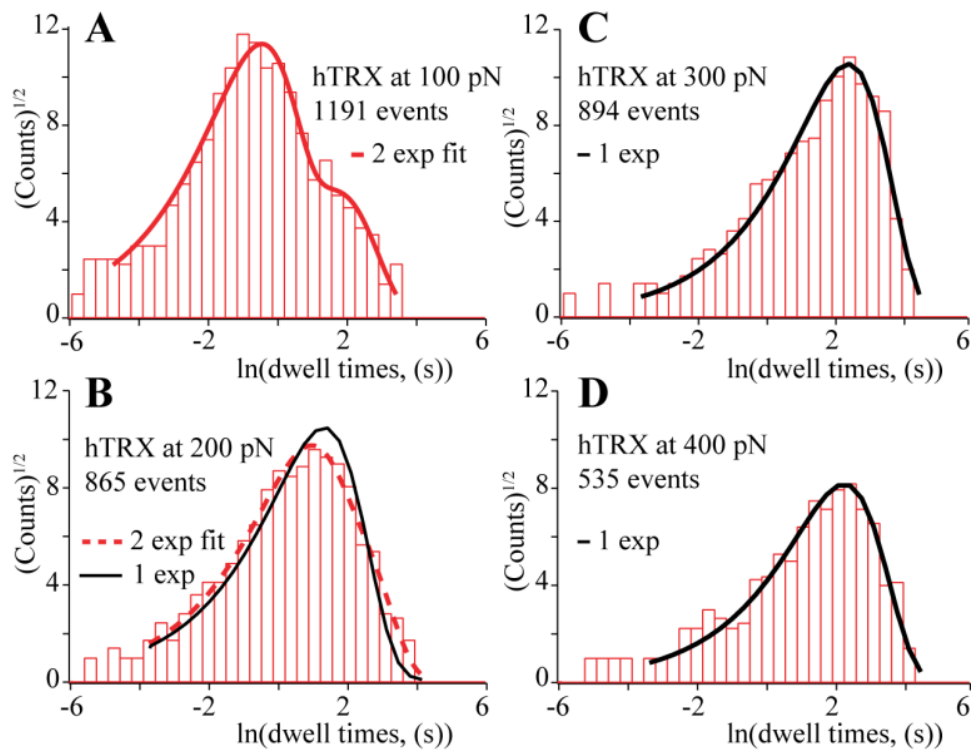

Figure 4. Dwell time histograms of disulfide reductions. Two processes are well resolvable at $100 \mathrm{pN}$ and $200 \mathrm{pN}$. One apparent process is adequate at $300 \mathrm{pN}$ and $400 \mathrm{pN}$. 


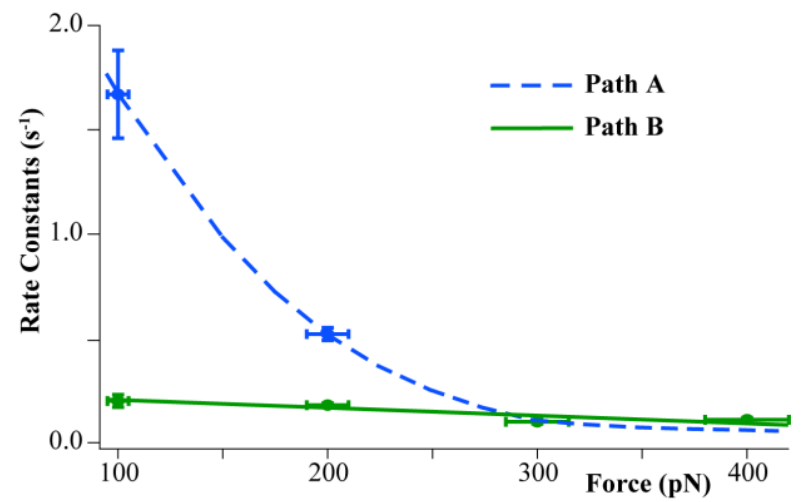

Figure 5. Disulfide reduction rate constants vs clamping force. Two disulfide reduction pathways, paths A and B, are elucidated from exponential fitting and dwell time analysis.

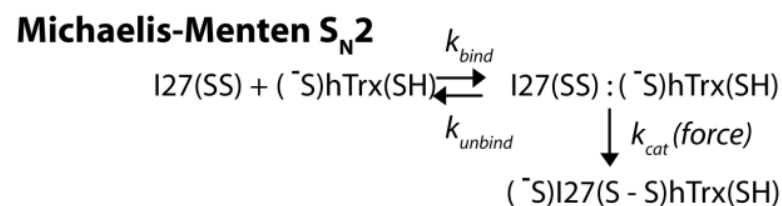

\section{Direct $\mathrm{S}_{\mathrm{N}} 2$}

( $\mathrm{S}) \mathrm{I} 27(\mathrm{~S}-\mathrm{S}) \mathrm{h} \operatorname{Trx}(\mathrm{SH})$

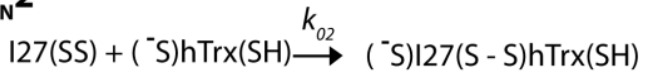

\section{E2 followed by $S_{N} 2$}

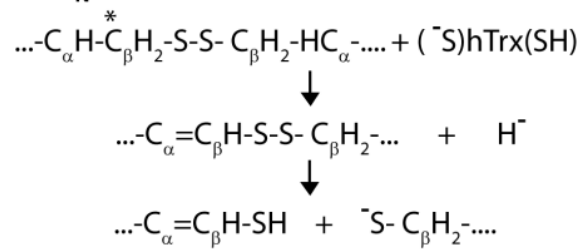

Scheme 1. Selected reaction schemes for a disulfide bond reduction by hTrx. In a force-dependent Michaelis-Menten disulfide reduction mechanism, which explains path $\mathrm{A}, \boldsymbol{k}_{\text {bind }}, \boldsymbol{k}_{\text {unbind }}$, and $\boldsymbol{k}_{\text {cat }}$ are pseudo-first order reaction rate constants. In a direct $S_{N} 2$ mechanism, one of the catalytic cysteines in $h$ Trx attacks a disulfide bond to be broken. A pseudo-first order reaction rate constant, $k_{02}$, is calculated as: $k_{02}=\gamma^{*}[\mathrm{hTrx}] * \exp \left(\right.$ Force $\left.^{*} \Delta x_{02} /\left(k_{B} T\right)\right)$, see text. In a schematic labeled "E2 followed by SN2", see text, a catalytic cysteine from within hTrx does not directly attack a disulfide bond to be broken. The symbols "C $\alpha$ " and "C $\beta$ " refer to $\alpha$-carbons and $\beta$-carbons in cysteines within I27. The star above one "C $\beta$ " signifies that $\beta$-carbons (either ones) are most likely targets for nucleophilic attack from catalytic cysteines within hTrx. After elimination, a hydride attacks the disulfide bond.

\section{Notes and references}

$\dagger$ Electronic Supplementary Information (ESI) is available: details of the data analysis and data fitting.

1 D. A. Kraut, K. S. Carroll, D. Herschlag, Annual Review of Biochemistry 2003, 72, 517-571.

2 S. Hammes-Schiffer, S. J. Benkovic, Annual Review of Biochemistry 2006, 75, 519-541. 
3 U. B. S. Rawat, A. V. Zavialov, J. Sengupta, M. Valle, R. A. Grassucci, J. Linde, B. Vestergaard, M. Ehrenberg, J. Frank, Nature 2003, 421, 87-90.

4 K. A. Henzler-Wildman, M. Lei, V. Thai, S. J. Kerns, M. Karplus, D. Kern, Nature 2007, 450, 913-927.

5 J. Villali, D. Kern, Current Opinion in Chemical Biology 2010, 14, 636-643.

6 A. P. Wiita, R. Perez-Jimenez, K. A. Walther, F. Grater, B. J. Berne, A. Holmgren, J. M. Sanchez-Ruiz, J. M. Fernandez, Nature 2007, 450, 124-130.

7 R. Perez-Jimenez, J. Y. Li, P. Kosuri, I. Sanchez-Romero, A. P. Wiita, D. Rodriguez-Larrea, A. Chueca, A. Holmgren, A. Miranda-Vizuete, K. Becker, S. H. Cho, J. Beckwith, E. Gelhaye, J. P. Jacquot, E. A. Gaucher, J. M. Sanchez-Ruiz, B. J. Berne, J. M. Fernandez, Nature Structural \& Molecular Biology 2009, 16, 592-599.

8 E. S. Arner, A. Holmgren, European Journal of Biochemistry 2000, 267, 6102-6109.

9 A. Holmgren, Annual Review of Biochemistry 1985, 54, 237-271.

10 R. Szoszkiewicz, S. R. K. Ainavarapu, A. P. Wiita, R. Perez-Jimenez, J. M. Sanchez-Ruiz, J. M. Fernandez, Langmuir 2008, 24, 1356-1364.

11 D. R. Cox, H. D. Miller, The theory of stochastic processes. Editor, Methuen, London, 1965.

12 O. P. Hamill, A. Marty, E. Neher, B. Sakmann, F. J. Sigworth, Pflugers Archiv-European Journal of Physiology 1981, 391 , $85-100$.

13 F. J. Sigworth, E. Neher, Nature 1980, 287, 447-449.

14 R. W. Aldrich, D. P. Corey, C. F. Stevens, Nature 1983, 306, 436-441.

15 F. J. Sigworth, S. M. Sine, Biophysical Journal 1987, 52, 1047-1054.

16 A. A. Carter, R. E. Oswald, Journal of Neuroscience Methods 1995, 60, 69-78.

17 R. E. Wachtel, Journal of Neuroscience Methods 1988, 25, 121-128.

18 A. P. Wiita, S. R. K. Ainavarapu, H. H. Huang, J. M. Fernandez, Proceedings of the National Academy of Sciences of the United States of America 2006, 103, 7222-7227.

19 M. Carrion-Vazquez, A. F. Oberhauser, S. B. Fowler, P. E. Marszalek, S. E. Broedel, J. Clarke, J. M. Fernandez, Proceedings of the National Academy of Sciences of the United States of America 1999, 96, 3694-3699.

20 X. L. Ren, M. Bjornstedt, B. Shen, M. L. Ericson, A. Holmgren, Biochemistry 1993, 32, 9701-9708.

21 H. J. Butt, B. Cappella, M. Kappl, Surface Science Reports 2005, 59, 1-152.

22 T. D. Li, J. P. Gao, R. Szoszkiewicz, U. Landman, E. Riedo, Phyical Review B 2007, 75, 115415.

23 R. Szoszkiewicz, A. J. Kulik, G. Gremaud, M. Lekka, Applied Physics Letters 2005, 86, 123901.

24 D. A. Koster, C. H. Wiggins, N. H. Dekker, Proceedings of the National Academy of Sciences of the United States of America 2006, 103, 1750-1755.

25 B. Efron, The Jackknife, the Bootstrap, and Other Resampling Plans. Editor, S.I.A.M., Philadelphia, PA, 1982.

26 P. A. Fernandes, M. J. Ramos, Chemistry-a European Journal 2004, 10, 257-266 DOI 10.1002/chem.200305343.

27 S. Garcia-Manyes, J. Liang, R. Szoszkiewicz, T. L. Kuo, J. M. Fernandez, Nature Chemistry 2009, 1, 236-242.

28 D. Jiao, M. Barfield, J. E. Combariza, V. J. Hruby, Journal of the American Chemical Society 1992, 114, 3639-3643.

29 R. A. Marcus, Pure and Applied Chemistry 1997, 69, 13-29.

30 C. S. Sevier, C. A. Kaiser, Nature Reviews Molecular Cell Biology 2002, 3, 836-847.

31 C. C. Page, C. C. Moser, X. X. Chen, P. L. Dutton, Nature 1999, 402, 47-52.

32 A. A. Voityuk, Journal of Physical Chemistry B 2011, 115, 12202-12207.

33 C. C. Moser, J. M. Keske, K. Warncke, R. S. Farid, P. L. Dutton, Nature 1992, 355, 796-802.

34 H. B. Gray, J. R. Winkler, Quarterly Reviews of Biophysics 2003, 36, 341-372.

35 Z. Y. Cheng, J. F. Zhang, D. P. Ballou, C. H. Williams, Chemical Reviews 2011, 111, 5768-5783.

36 A. Dey, R. Szoszkiewicz, Nanotechnology 2012, 23, 175101.

37 I. T. Suydam, C. D. Snow, V. S. Pande, S. G. Boxer, Science 2006, 313, 200-204.

38 N. M. Giles, G. I. Giles, C. Jacob, Biochemical and Biophysical Research Communications 2003, 300, 1-4.

39 G. I. Giles, C. Jacob, Biological Chemistry 2002, 383, 375-388. 


\section{Supplementary Materials for "Single-molecule studies of disulfide bond reduction......." by $R$. Szoszkiewicz}

\section{Detailed analyses of single molecule disulfide reduction events in $\left(\text { I27 }_{\mathrm{Ss}}\right)_{8}$ by human thioredoxin (hTRX).}

Tables S1 to S4 summarize the disulfide reduction rate constants obtained via two methods used in the paper: exponential fitting and dwell time analysis. Each table contains the results of fitting the experimental data at a given clamping force with one and two rate constants. Number of single disulfide reduction events used at each case is explicitly noted as the "number of reductions". After each table a corresponding plot of dwell time histograms is provided.

"Ensemble Average" means exponential fitting to an averaged FC-AFM length trace at a given clamping force. FC-AFM length traces represent temporal changes of the protein end-toend length during a disulfide reduction phase only. Errors of the rate constants are calculated by bootstrapping as explained in the paper.

"Log. Hist." means dwell time analysis of disulfide reduction events by means of logarithmic histograms. Dwells are calculated from the FC-AFM length traces as described in the paper. Two ways are used to obtain the rate constants via this method. First, the rate constants are obtained by directly fitting the pdf function for each histogram without any assumptions. These results are presented in a column labeled "Log. Hist. direct". The pdf function $g(x)=\Sigma_{i} a_{i} \exp \left(x+\ln \left(k_{i}\right)+\right.$ $\left.\delta x / 2+\exp \left(x+\ln \left(k_{i}\right)+\delta x / 2\right)\right)$, where $\Sigma_{i}$ sums over fitted reduction rate constants $k_{i}, x=\ln (t)$ is a natural logarithm of time $t, \delta x$ is a bin size, and $a_{i}$ is a fraction of a total number of events $N$. See also the Materials and Methods in the paper. Next, one of the rate constants is constrained to be $0.1 \mathrm{~s}^{-1}$ across all clamping forces and these results are within a column "Log. Hist. constrained". The value of $0.1 \mathrm{~s}^{-1}$ comes from unconstrained fits at $400 \mathrm{pN}$ clamping force, where disulfide reductions at this rate constant become dominant. Time resolution of $1 \mathrm{~ms}$ for all the fits was used. 
Table S1. Disulfide reduction rate constants obtained at $100 \mathrm{pN}$ clamping force. Results: processes with two rate constants have best $\chi_{\text {red }}^{2}$ and very well defined rates and amplitudes.

\begin{tabular}{|c|l|l|l|}
\hline $\begin{array}{c}\text { hTRX @ } 100 \mathrm{pN} \\
\mathbf{1 1 9 1} \text { reductions }\end{array}$ & $\begin{array}{l}\text { Ensemble } \\
\text { Average }\end{array}$ & $\begin{array}{l}\text { Log. Hist. } \\
\text { direct }\end{array}$ & $\begin{array}{l}\text { Log. Hist. } \\
\text { constrained }\end{array}$ \\
\hline $\begin{array}{c}\text { Single rate } \\
\text { constant }\left(\mathrm{s}^{-1}\right)\end{array}$ & $1.10 \pm 0.08$ & $1.10 \pm 0.03$ & N/A \\
\hline$\chi_{\text {red }}^{2}$ & 11 & 13 & N/A \\
\hline \multicolumn{2}{|c|}{ Two rate constants } \\
\hline rate constant $_{1}\left(\mathrm{~s}^{-1}\right)$ & $0.20 \pm 0.03$ & $0.20 \pm 0.03$ & 0.10 \\
\hline $\begin{array}{c}\text { amplitude }_{1} \\
{[\% \text { of total }]^{-1}}\end{array}$ & $\begin{array}{l}12.1 \pm 0.1 \\
{[19 \%]}\end{array}$ & $\begin{array}{l}223 \pm 31 \\
{[19 \%]}\end{array}$ & $\begin{array}{l}132 \pm 14 \\
{[11 \%]}\end{array}$ \\
\hline rate constant $_{2}\left(\mathrm{~s}^{-1}\right)$ & $1.67 \pm 0.10$ & $1.69 \pm 0.10$ & $1.69 \pm 0.10$ \\
\hline $\begin{array}{c}\text { amplitude }_{2} \\
{[\% \text { of total }]^{2}}\end{array}$ & $\begin{array}{l}53.0 \pm 0.1 \\
{[81 \%]}\end{array}$ & $968 \pm 31$ & $\begin{array}{l}968 \pm 31 \\
{[81 \%]}\end{array}$ \\
\hline$\chi_{\text {red }}^{2}$ & 0.4 & 0.4 & 3.9 \\
\hline
\end{tabular}

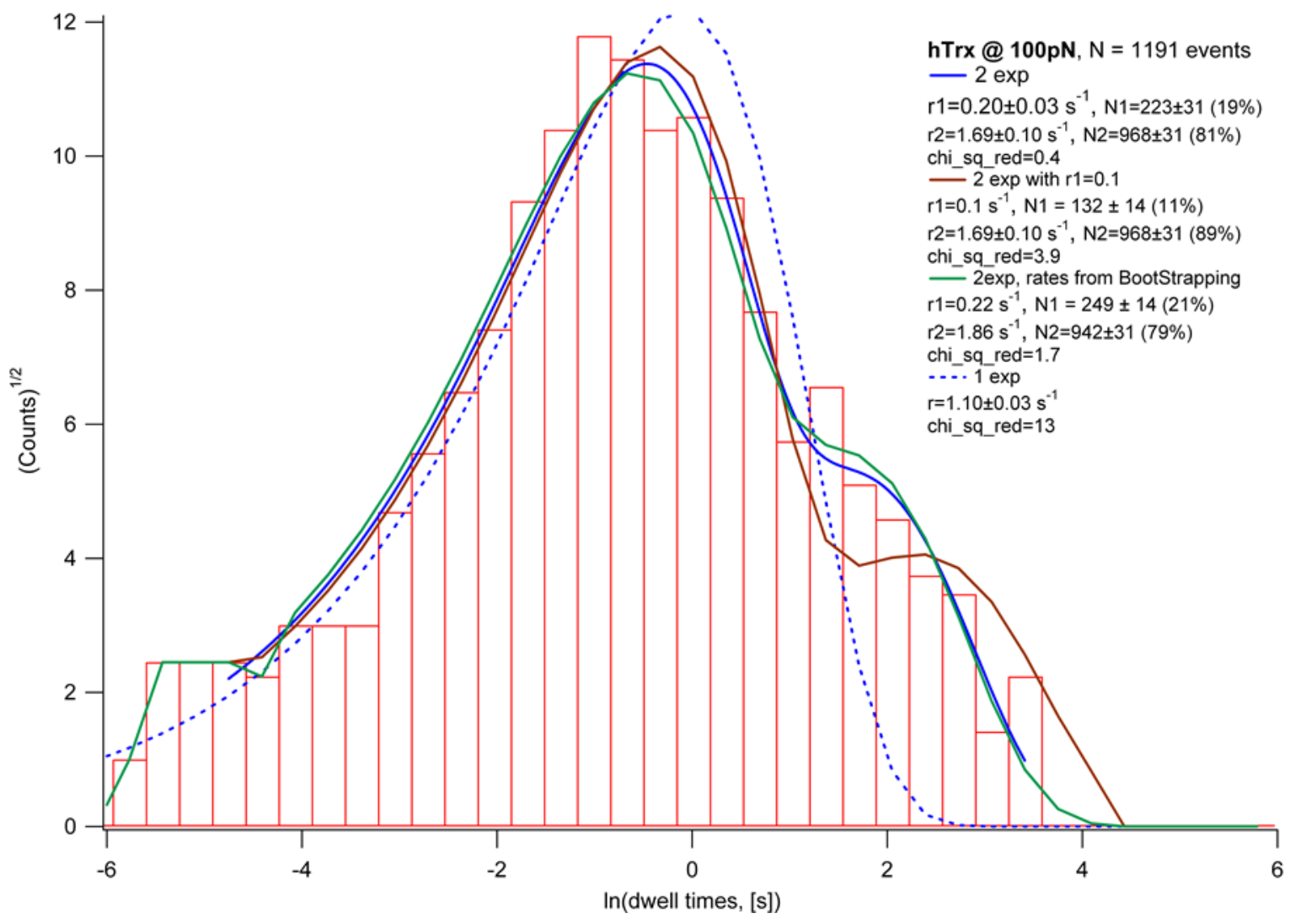


Table S2. Disulfide reduction rate constants obtained at $200 \mathrm{pN}$ clamping force. Conclusions are similar as in the case of Table S1.

\begin{tabular}{|c|c|c|c|}
\hline $\begin{array}{l}\text { hTRX@200 pN } \\
865 \text { reductions }\end{array}$ & $\begin{array}{l}\text { Ensemble } \\
\text { Average }\end{array}$ & $\begin{array}{l}\text { Log. Hist. } \\
\text { direct }\end{array}$ & $\begin{array}{l}\text { Log. Hist. } \\
\text { constrained }\end{array}$ \\
\hline $\begin{array}{l}\text { Single rate } \\
\text { constant }\left(\mathrm{s}^{-1}\right)\end{array}$ & $0.31 \pm 0.02$ & $0.28 \pm 0.01$ & $\mathrm{~N} / \mathrm{A}$ \\
\hline$\chi_{\text {red }}^{2}$ & 3.6 & 3.3 & $\mathrm{~N} / \mathrm{A}$ \\
\hline \multicolumn{4}{|c|}{ Two rate constants } \\
\hline rate constant ${ }_{1}\left(\mathrm{~s}^{-1}\right)$ & $0.18 \pm 0.04$ & $0.15 \pm 0.02$ & 0.10 \\
\hline amplitude $_{1}$ & $33.2 \pm 0.1$ & $321 \pm 81$ & $146 \pm 25$ \\
\hline [\% of total] & {$[46 \%]$} & [37\%] & {$[17 \%]$} \\
\hline rate constant ${ }_{2}\left(\mathrm{~s}^{-1}\right)$ & $0.54 \pm 0.21$ & $0.49 \pm 0.06$ & $0.38 \pm 0.02$ \\
\hline amplitude $_{2}$ & $38.7 \pm 0.1$ & $544 \pm 81$ & $720 \pm 25$ \\
\hline$[\%$ of total $]$ & {$[54 \%]$} & {$[63 \%]$} & {$[83 \%]$} \\
\hline$\chi_{\text {red }}^{2}$ & 0.7 & 0.9 & 1.2 \\
\hline
\end{tabular}

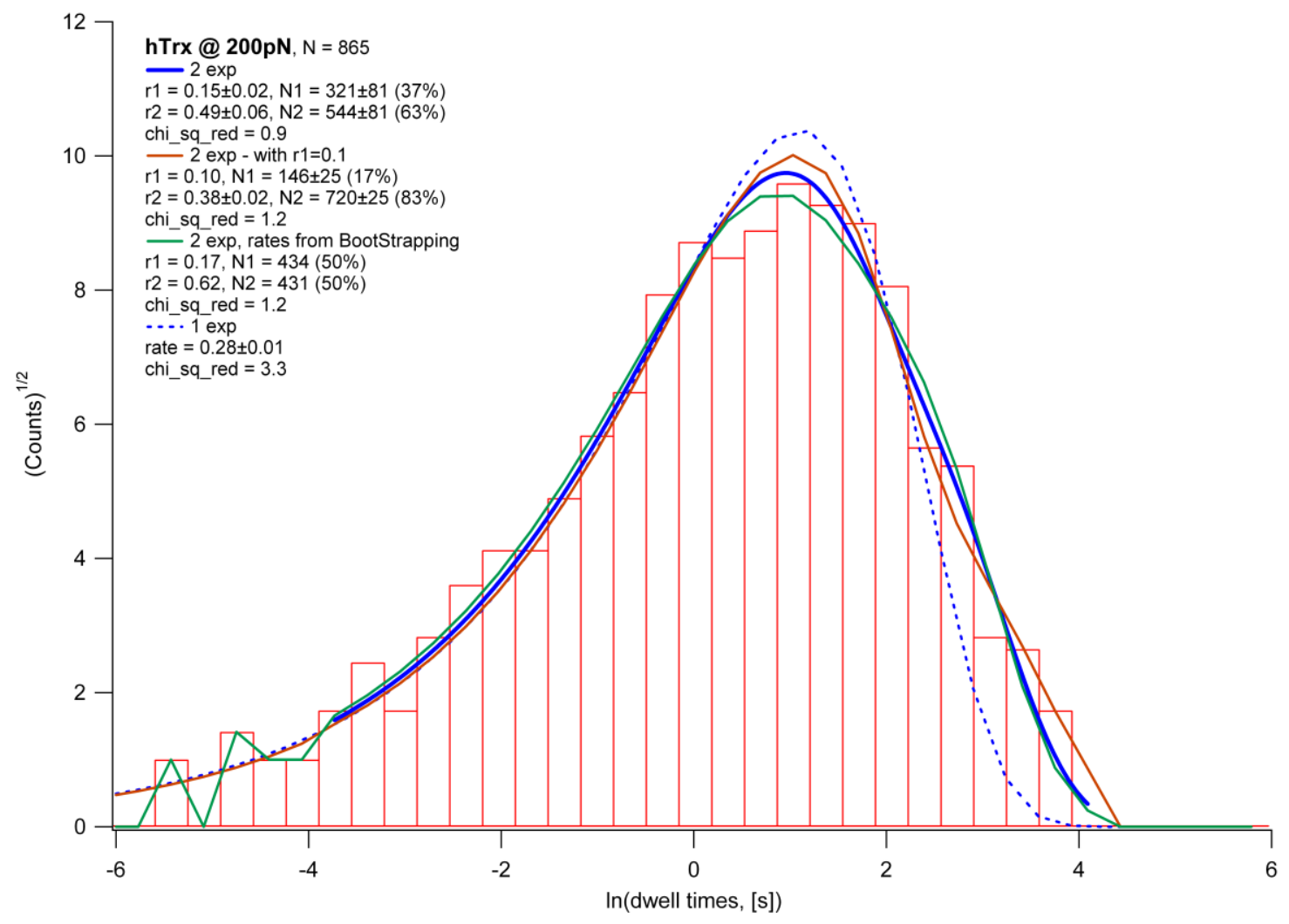


Table S3. Disulfide reduction rate constants obtained at 300pN clamping force. Results: processes with two rate constants have better $\chi_{\text {red }}^{2}$ than the ones with a single reaction rate constant, but a second rate constant is not well defined and applies to a low number of events.

\begin{tabular}{|c|l|l|l|}
\hline $\begin{array}{l}\text { hTRX @ } 300 \mathrm{pN} \\
\text { 894 reductions }\end{array}$ & $\begin{array}{l}\text { Ensemble } \\
\text { Average }\end{array}$ & $\begin{array}{l}\text { Log. Hist. } \\
\text { direct }\end{array}$ & $\begin{array}{l}\text { Log. Hist. } \\
\text { constrained }\end{array}$ \\
\hline $\begin{array}{c}\text { Single rate } \\
\text { constant }\left(\mathrm{s}^{-1}\right)\end{array}$ & $0.10 \pm 0.01$ & $0.09 \pm 0.01$ & N/A \\
\hline$\chi_{\text {red }}^{2}$ & 1.2 & 1.7 & N/A \\
\hline \multicolumn{4}{|c|}{ Two rate constants } \\
\hline rate constant ${ }_{1}\left(\mathrm{~s}^{-1}\right)$ & $0.09 \pm 0.01$ & $0.09 \pm 0.01$ & 0.10 \\
\hline $\begin{array}{c}\text { amplitude }_{1} \\
{[\% \text { of total }]}\end{array}$ & $\begin{array}{l}60.7 \pm 0.1 \\
{[94 \%]}\end{array}$ & $\begin{array}{l}847 \pm 22 \\
{[94 \%]}\end{array}$ & $\begin{array}{l}867 \pm 17 \\
{[97 \%]}\end{array}$ \\
\hline rate constant ${ }_{2}\left(\mathrm{~s}^{-1}\right)$ & $2.9 \pm 1.7$ & $1.1 \pm 0.6$ & $1.6 \pm 1.4$ \\
\hline $\begin{array}{c}\text { amplitude } \\
{[}\end{array}$ & $\begin{array}{l}3.9 \pm 0.1 \\
{[6 \%]}\end{array}$ & $51 \pm 22$ & $51 \pm 22$ \\
{$[6 \%]$} & {$[3 \%]$} \\
\hline$\chi_{\text {red }}^{2}$ & 0.8 & 1.2 & 1.7 \\
\hline
\end{tabular}

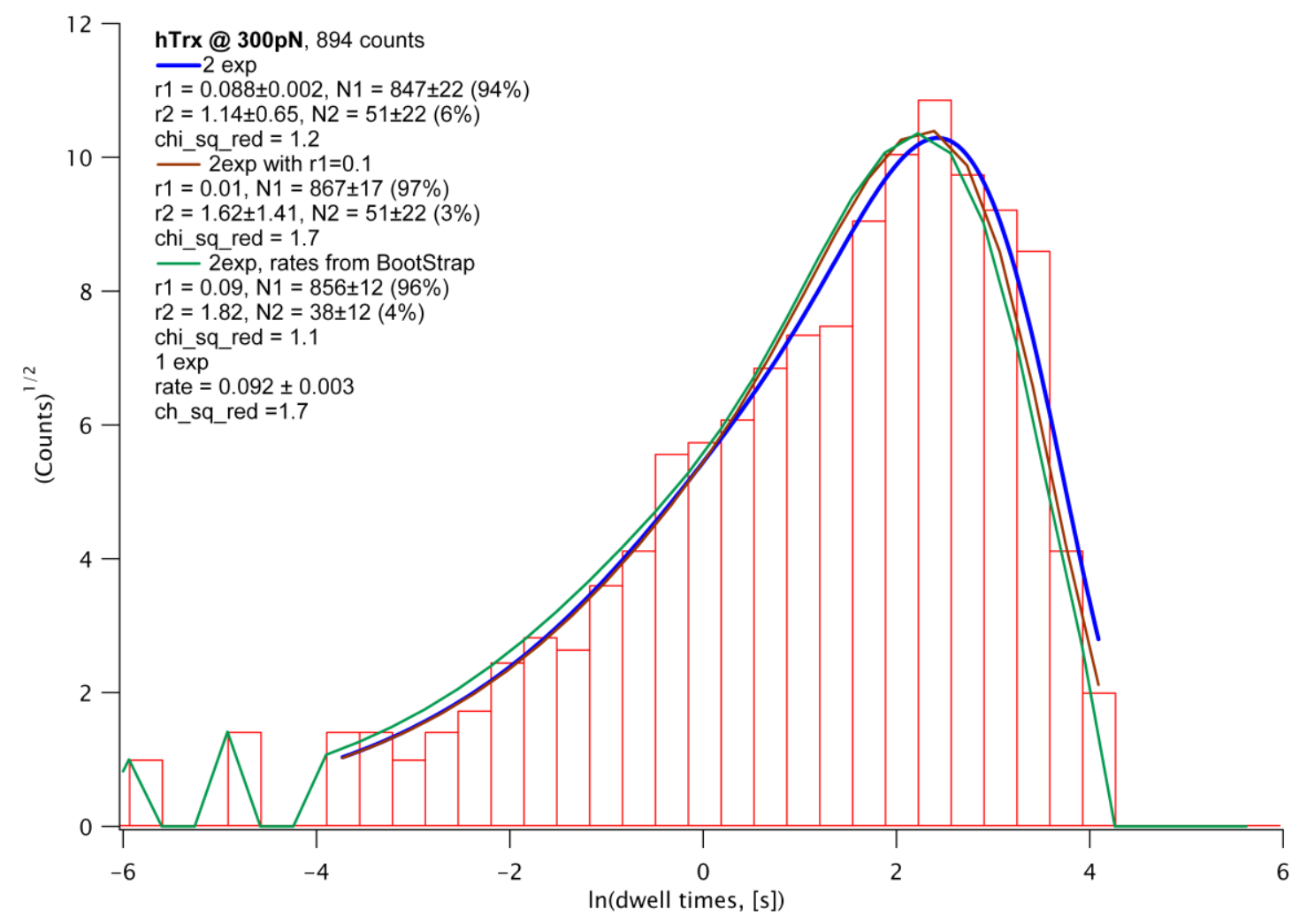


Table S4. Disulfide reduction rate constants obtained at $400 \mathrm{pN}$ clamping force. Conclusions are similar as in the case of Table S3.

\begin{tabular}{|c|c|c|c|}
\hline $\begin{array}{l}\text { hTRX@400 pN } \\
535 \text { reductions }\end{array}$ & $\begin{array}{l}\text { Ensemble } \\
\text { Average }\end{array}$ & $\begin{array}{l}\text { Log. Hist. } \\
\text { direct }\end{array}$ & $\begin{array}{l}\text { Log. Hist. } \\
\text { constrained }\end{array}$ \\
\hline $\begin{array}{c}\text { Single rate } \\
\text { constant }\left(\mathrm{s}^{-1}\right)\end{array}$ & $0.12 \pm 0.01$ & $0.11 \pm 0.01$ & $\mathrm{~N} / \mathrm{A}$ \\
\hline$\chi_{\text {red }}^{2}$ & 0.9 & 1.2 & $\mathrm{~N} / \mathrm{A}$ \\
\hline \multicolumn{4}{|c|}{ Two rate constants } \\
\hline rate constant $1\left(\mathrm{~s}^{-1}\right)$ & $0.11 \pm 0.03$ & $0.10 \pm 0.01$ & $\mathrm{~N} / \mathrm{A}$ \\
\hline $\begin{array}{l}\text { amplitude }_{1} \\
{[\% \text { of total }]}\end{array}$ & $\begin{array}{l}76.4 \pm 0.1 \\
{[93 \%]}\end{array}$ & $\begin{array}{l}507 \pm 41 \\
{[95 \%]}\end{array}$ & $\mathrm{N} / \mathrm{A}$ \\
\hline rate constant $2\left(\mathrm{~s}^{-1}\right)$ & $0.62 \pm 0.62$ & $0.63 \pm 0.63$ & $\mathrm{~N} / \mathrm{A}$ \\
\hline $\begin{array}{l}\text { amplitude }_{2} \\
{[\% \text { of total }]}\end{array}$ & $\begin{array}{l}6.2 \pm 0.1 \\
{[7 \%]}\end{array}$ & $\begin{array}{l}28 \pm 21 \\
{[5 \%]}\end{array}$ & N/A \\
\hline$\chi_{\text {red }}^{2}$ & 0.4 & 0.4 & $\mathrm{~N} / \mathrm{A}$ \\
\hline
\end{tabular}

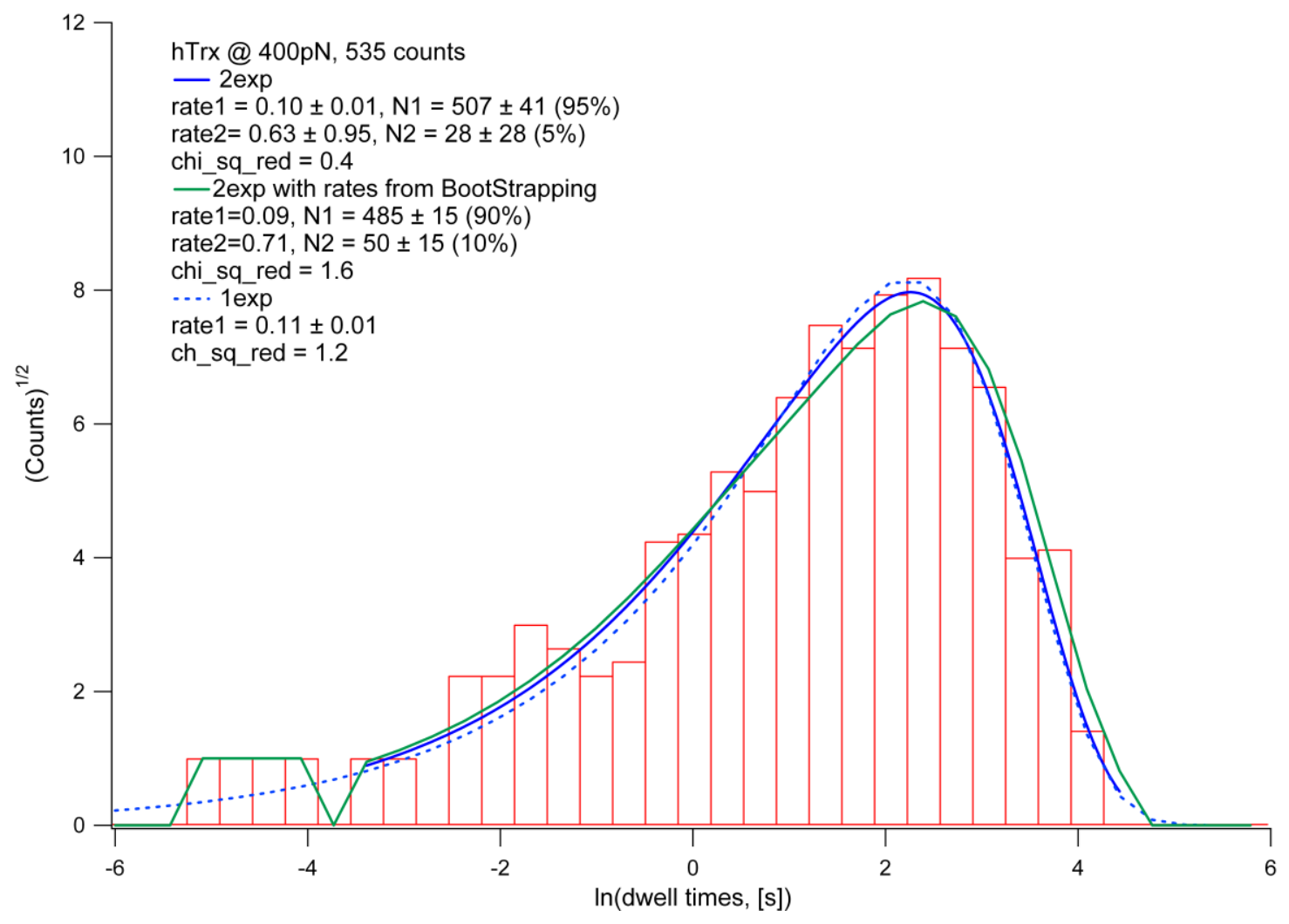




\section{Fitting parameters for a direct $S_{N} 2$ mechanism for path $B$}

Linear fits to the natural logarithm of rate constants (in $\mathrm{s}^{-1}$ ) obtained for path $\mathrm{B}$ are performed to get the parameters $\gamma$ and $\Delta x_{02}$ defined by the equation $k_{02}=\gamma^{*}[\operatorname{Trx}]^{*} \exp \left(F^{*} \Delta x_{02} /\left(k_{B} T\right)\right)$, where $k_{02}$ represents the rate constants for path $\mathrm{B}$.

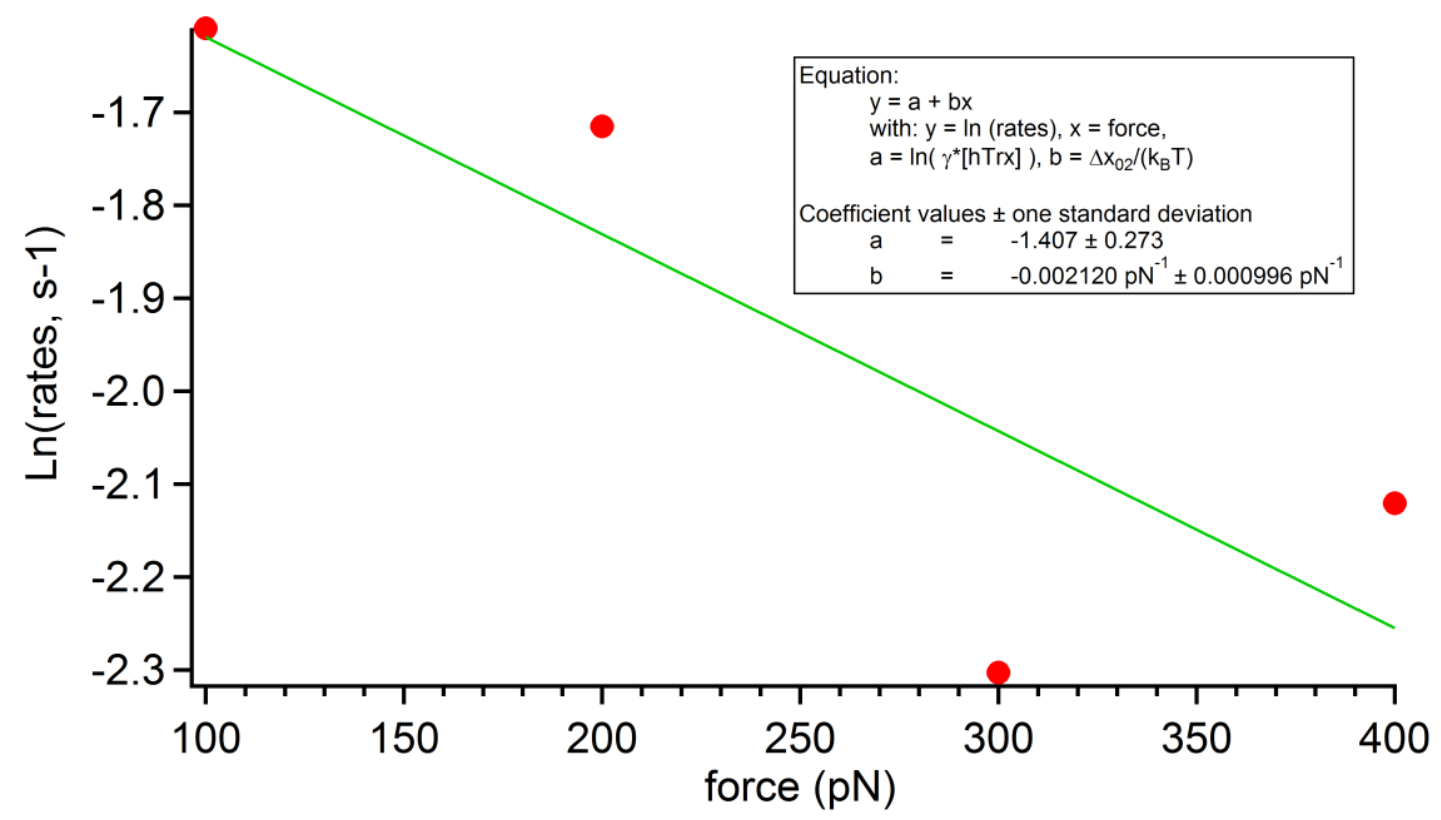

\section{Applying electron tunnelling mechanism for path $B$}

Path B could also relate to electron tunnelling. ${ }^{1}$ Tunnelling involves no bond rupture, no energy barrier, and hence no transition state. ${ }^{2}$ There is, however, a reorganization energy barrier is involved in ET, since the system must prepare by obtaining reactive radicals. Here, the ET process is likely to proceed via a thiyl radical (-S.) in one of the a catalytic cysteines and a subsequent electron transfer to a disulfide bond in $\left(\mathrm{I} 27_{\mathrm{SS}}\right)_{8}$. Then, the disulfide reduction itself proceeds via a thyil radial and a thiolate $\left(-\mathrm{S}^{-}\right){ }^{3}$ Formation of a thyil radial and a thiolate breaks open the disulfide bond, which is then separated by mechanical force. A rate constant for electron tunnelling, $k_{E T}$, is usually described be a semi-classical theory for a non-adiabatic electron transfer using a following formula: ${ }^{2} k_{E T}=(4 \pi / h) * V^{2} *\left(4 \pi \lambda k_{B} T\right)^{-1 / 2} * \exp [-(\Delta G$ $\left.+\lambda)^{2} /\left(4 \lambda k_{B} T\right)\right]$. Here: $h$ is the Planck's constant, $k_{B}$ is the Boltzmann's constant, $T$ is absolute temperature, $\lambda$ is a reorganization energy barrier, $V$ is a coupling potential 
between electronic orbitals of donor and acceptor, and $\Delta G$ is a driving energy. Due to substantial difficulty in estimating a value of $V$ in proteins, $k_{E T}$ is often approximated by a phenomenological Moser-Datton equation:

$$
\log \left(k_{E T}\right) \cong 13-0.6\left(R_{D A}-3.6\right)-3.1\left[(\Delta G+\lambda)^{2} / \lambda\right] \quad \text { Eq. } \mathrm{S} 1 .
$$

In Eq. $\mathrm{S} 1$ a decimal logarithm of $k_{E T}$ is used, $\Delta G$ and $\lambda$ are in $\mathrm{eV}$, and a donoracceptor distance $R_{D A}$ is in $\AA^{4-5}$ To estimate the value of $k_{E T}$ from Eq. S1, each of important parameters, i.e., $R_{D A}, \lambda$, and $\Delta G$, is estimated and discussed.

Using Eq. S1 one can estimate a high limit of $k_{E T}$. A maximum cut-off for the value of $R_{D A}$ is arbitrary estimated as $10 \AA$. Likely, substantially smaller values of $R_{D A}$ than $10 \AA$ are experimentally plausible. For example, a distance of a typical -S-S- bond is about $2 \AA$, and a hTrx catalytic groove is $5 \AA$ deep. ${ }^{1}$ However, $R_{D A}$ of less than $10 \AA$ would produce extremely high tunnelling rates, see below. Even the fastest tunnelling still needs to overcome a reorganizational energy barrier $\lambda$ of the order of available thermal energy, $k_{B} T$, i.e., $\lambda \sim k_{B} T=0.026 \mathrm{eV}$. Thus, supposing no extra driving energy, i.e., $\Delta G=0, \lambda \sim k_{B} T=0.026 \mathrm{eV}$, and $R_{D A}=10 \AA$, the value of $k_{E T}$ from Eq. $\mathrm{S} 1$ is of the order of $10^{9} \mathrm{~s}^{-1}$. Using $R_{D A}=1 \AA$ one obtains $k_{E T} \sim 10^{14} \mathrm{~s}^{-1}$. Those high tunnelling rate constant are typical in proteins, and particularly in enzymatic catalysis. ${ }^{6-7}$ To get $k_{E T}$ of the order of experimental values of $0.1 \mathrm{~s}^{-1}$, a substantial reorganizational energy barrier $\lambda$ needs to be accounted for.

Due to excess of NADPH used here to maintain hTrx in a reduced form the value of $\lambda$ will relate to formation of a thiyl radial in a one of the catalytic cysteines in $\mathrm{hTrx}$. Using density functional methods bond dissociation energy (BDE) of $81.7 \mathrm{kcal} / \mathrm{mol}$, or $3.5 \mathrm{eV}$, was obtained for an S-H bond. ${ }^{8}$ This S-H BDE value in a catalytic hTrx groove must be, however, lower than $3.5 \mathrm{eV}$. For example, an activation energy barrier of 86.1 
$\mathrm{kJ} / \mathrm{mol}$ or $0.89 \mathrm{eV}$ was found for a direct $\mathrm{S}_{\mathrm{N}} 2$ type disulfide reduction in (I27 $\left.{ }_{\mathrm{ss}}\right)_{8}$ by small molecules like hydroxide anion or tris(2-carboxyethyl)phosphine. ${ }^{9}$ Thus, for tunnelling to be competitive with a direct $\mathrm{S}_{\mathrm{N}} 2$ mechanism $\lambda \sim 1 \mathrm{eV}$ is expected. In fact, numerous studies reported that $\lambda$ in proteins is between 0.5 to $1.5 \mathrm{eV} .{ }^{7}{ }^{10}$ Using $R_{D A}=10 \AA$ and $\lambda=1.5 \mathrm{eV}$, Eq. $\mathrm{S} 1$ yields that a minimum $\Delta G$ of $0.7 \mathrm{eV}$ is needed to achieve electron tunnelling at $0.1 \mathrm{~s}^{-1}$. It is showed below that such a value of $\Delta G$ seems too high in the case of $h \operatorname{Tr} x$.

The value of $\Delta G$ depends on a protonation state of cysteine residues as well as orientation of polar groups and water molecules in the vicinity of the redox sites. ${ }^{11-12}$ Thus, $\Delta G$ is estimated from the difference between a redox potential of cysteine in water, e.g., at high dielectric constant environment, and a redox potential of a catalytic cysteine within hTrx, i.e., at low dielectric constant environment. Redox potentials for several eukaryotic thioredoxins have been carefully measured to be about $-0.3 \mathrm{eV} .^{13} \mathrm{~A}$ redox potential for a disulfide bond is estimated between $-0.2 \mathrm{eV}$ from the studies with glutathione, to $-0.1 \mathrm{eV}$ from the studies with some cysteine pairs. ${ }^{3,14}$ Thus, the value of $\Delta G$ is estimated between 0.1 to $0.2 \mathrm{eV}$. Using $R_{D A}=10 \AA, \lambda=1.5 \mathrm{eV}$ and $\Delta G=0.1 \mathrm{eV}$ Eq. S1 yields a tunnelling rate constant of $7 * 10^{3} \mathrm{~s}^{-1}$, which decreases to $2 * 10^{3} \mathrm{~s}^{-1}$ when $\Delta G=0.2 \mathrm{eV}$.

However, at $1,000 \mathrm{~s}^{-1}$ tunnelling rate constants hTrx diffusion might start to control the ET process. This issue is addressed as follows. Since $\left(\mathrm{I} 27_{\mathrm{SS}}\right)_{8}$ is clamped, and hence does not diffuse appreciably, a mean time between collisions of hTrx with (I27ss) 8 is roughly twice the mean time, $\tau$, between collision of two hTrx molecules. Due to a low hTrx concentration of $10 \mu \mathrm{M}$, a value of $\tau$ is estimated from a ratio of a mean free path, $l$, to an RMS velocity, $v_{\mathrm{RMS}}$, of a hTrx molecule. The value of $l=\left(\pi n d^{2}\right)^{-1}$, where $n$ is 
concentration of hTrx molecules and $d$ is a mean diameter of a hTrx molecule. The value of $v_{\mathrm{RMS}}=\left(3 k_{B} T / m\right)$, where $m$ is a mass of a hTrx molecule. Using hTrx molecular mass of $12 \mathrm{kDa}, \tau$ of the order of one $\mu$ s are obtained at room temperature. Consequently, hTrx diffusion should not influence tunnelling.

Overall, the ET rate constants are two orders of magnitude larger than what is possible to measure using current FC-AFM setups. ${ }^{15}$ Thus, while electron tunnelling cannot be excluded, its experimental verification is expected to involve FC-AFM spectrometers with much higher bandwidth.

\section{REFERENCES}

1. Perez-Jimenez, R.; Li, J. Y.; Kosuri, P.; Sanchez-Romero, I.; Wiita, A. P.; Rodriguez-Larrea, D.; Chueca, A.; Holmgren, A.; Miranda-Vizuete, A.; Becker, K.; Cho, S. H.; Beckwith, J.; Gelhaye, E.; Jacquot, J. P.; Gaucher, E. A.; Sanchez-Ruiz, J. M.; Berne, B. J.; Fernandez, J. M., Diversity of chemical mechanisms in thioredoxin catalysis revealed by single-molecule force spectroscopy (vol 16, pg 890, 2009). Nature Structural \& Molecular Biology 2009, 16, 1331-1331.

2. Marcus, R. A., Electron transfer reactions in chemistry. Theory and experiment. Pure and Applied Chemistry 1997, 69, 13-29.

3. Sevier, C. S.; Kaiser, C. A., Formation and transfer of disulphide bonds in living cells. Nature Reviews Molecular Cell Biology 2002, 3, 836-847.

4. Page, C. C.; Moser, C. C.; Chen, X. X.; Dutton, P. L., Natural engineering principles of electron tunnelling in biological oxidation-reduction. Nature 1999, 402, 4752.

5. Voityuk, A. A., Long-Range Electron Transfer in Biomolecules. Tunneling or Hopping? Journal of Physical Chemistry B 2011, 115, 12202-12207.

6. Moser, C. C.; Keske, J. M.; Warncke, K.; Farid, R. S.; Dutton, P. L., Nature of Biological Electron-Transfer. Nature 1992, 355, 796-802.

7. Gray, H. B.; Winkler, J. R., Electron tunneling through proteins. Quarterly Reviews of Biophysics 2003, 36, 341-372.

8. Himo, F.; Siegbahn, P. E. M., Quantum chemical studies of radical-containing enzymes. Chemical Reviews 2003, 103, 2421-2456. 
9. Garcia-Manyes, S.; Liang, J.; Szoszkiewicz, R.; Kuo, T. L.; Fernandez, J. M., Force-activated reactivity switch in a bimolecular chemical reaction. Nature Chemistry 2009, 1, 236-242.

10. Moser, C. C.; Farid, T. A.; Chobot, S. E.; Dutton, P. L., Electron tunneling chains of mitochondria. Bba-Bioenergetics 2006, 1757, 1096-1109.

11. Gao, J. A.; Muller, P.; Wang, M.; Eckhardt, S.; Lauz, M.; Fromm, K. M.; Giese, B., Electron Transfer in Peptides: The Influence of Charged Amino Acids. Angewandte Chemie International Edition 2011, 50, 1926-1930.

12. Warshel, A.; Sharma, P. K.; Kato, M.; Xiang, Y.; Liu, H. B.; Olsson, M. H. M., Electrostatic basis for enzyme catalysis. Chem Rev 2006, 106, 3210-3235.

13. Cheng, Z. Y.; Zhang, J. F.; Ballou, D. P.; Williams, C. H., Reactivity of Thioredoxin as a Protein Thiol-Disulfide Oxidoreductase. Chemical Reviews 2011, 111, 5768-5783.

14. All potentials are related to the Standard Hydrogen Electrode.

15. Dey, A.; Szoszkiewicz, R., Complete noise analysis of a simple force spectroscopy AFM setup and its applications to study nanomechanics of mammalian Notch 1 protein. Nanotechnology 2012, 23, 175101. 